\title{
Vaccinating SIS epidemics under evolving perception in heterogeneous networks
}

\author{
Xiao-Jie $\mathrm{Li}^{1}$ and Xiang $\mathrm{Li}^{1,2,3, \mathrm{a}}$ \\ ${ }^{1}$ Adaptive Networks and Control Lab, Department of Electronic Engineering - Fudan University, Shanghai 200433, \\ P.R. China \\ ${ }^{2}$ Research Center of Smart Networks and Systems, School of Information Science and Engineering - \\ Fudan University, Shanghai 200433, P.R. China \\ ${ }^{3}$ MOE Frontiers Center for Brain Science, Institutes of Brain Science - Fudan University, Shanghai 200433, \\ P.R. China
}

Received 13 July 2020 / Received in final form 10 August 2020 / Accepted 20 August 2020

Published online 5 October 2020

(C) EDP Sciences / Società Italiana di Fisica / Springer-Verlag GmbH Germany, part of Springer Nature, 2020

\begin{abstract}
Vaccination is an effective intervention against epidemics. Previous work has demonstrated that psychological cognition affects individual behavior. However, perceptual differences between individuals, as well as the dynamics of perceptual evolution, are not taken into account. In order to explore how these realistic characteristics of psychological cognition influence collective vaccination behavior, we propose a prospect theory based evolutionary vaccination game model, where the evolution of reference points is used to characterize changes in perception. We compare the fractions of vaccinated individuals and infected individuals under variable reference points with those under the expected utility theory and the fixed reference point, and highlight the role of evolving perception in promoting vaccination and contributing to epidemic control. We find that the epidemic size under variable reference point is always less than that under the expected utility theory. Finding that there exists a vaccination cost threshold for the cognitive effect, we develop a novel mixed-reference-point mechanism by combining individual psychological characteristics with network topological feature. The effectiveness of this mechanism in controlling the network epidemics is verified with numerical simulations. Compared with pure reference points, the mixed-reference-point mechanism can effectively reduce the final epidemic size, especially at a large vaccination cost.
\end{abstract}

\section{Introduction}

The outbreak of the COVID-19 epidemic has not only threatened public health but also seriously influenced almost every facet of lives [1]. Developing effective vaccines is the only long-term solution to this epidemic [2]. Since a vaccinated individual can not only protect himself from being infected but also his unvaccinated neighbors, vaccination can be regarded as an altruistic cooperative behavior. However, self-interested individuals expect to benefit from the vaccination behavior of others, which leads to vaccination being a social dilemma [3,4]. Wang et al. [5] and Perc et al. [6] reviewed rich researches on studying the complex interactions between vaccination behavior and epidemic dynamics. Moreover, many studies [7-12] have explored the factors that affect individual vaccination behavior. For example, Fu et al. [8] investigated the role of individual imitation behavior and network structure in vaccination by integrating an epidemiological process into a simple agent-based model of

\footnotetext{
${ }^{\mathrm{a}}$ e-mail: lix@fudan.edu.cn
}

adaptive learning. Zhang et al. [9] addressed the impact of the more rational decision-making of individuals on voluntary vaccination, where the memory effect was considered. Buonomo [12] studied the impact of available information about the spreading of a coronavirus disease (COVID-19) on vaccination coverage. However, such efforts fail to account for cognitive effects that are claimed to be important factors in determining individual behavior [13-15]. Furthermore, many psychological and behavioral experiments show that individuals are bounded rationality and bounded cognition [16]. Although there is no vaccine available for the COVID-19 epidemic at the time of writing this paper, the development of a vaccine is proceeding rapidly, and understanding how individual psychological cognition affects vaccination decision-making is of great significance to epidemic control.

Game theory has been widely used to study the problem of epidemic transmission and control $[17,18]$. In the existing game-theoretic studies, individuals are commonly assumed to be risk-neutral and make decisions based on the expected utility theory. However, the expected utility theory cannot explain some anomalies of preferences, such 
as Allais Paradox and Ellsberg Paradox [19]. In order to model and explain the decisions of individuals under risk and uncertainty, Kahneman and Tversky [20] proposed the Nobel Prize-winning prospect theory, which has been applied in many fields [21-23]. In the epidemic spreading process, the risk mainly comes from the interaction between individuals. Hota et al. [23] examined the impact of probability perception on the Nash equilibrium protection strategies. Li et al. [24] modeled individual behavior by utilizing the weighting effect and framing effect of the prospect theory, respectively, and analyzed the impact of different perceptions on vaccination. However, perceptual differences between individuals and the dynamics of perceptual evolution are not taken into account. In reality, as the epidemics change rapidly, individual perception also changes from time to time, which motivates us to explore the influence of evolving perception on individual vaccination decision and epidemic transmission.

An individual perceives the payoff either as a gain or a loss based on a reference point, showing the characteristic of reference dependence, which is the core of the prospect theory $[20,25]$. Therefore, we use variable reference points to capture the individual perceptual evolution. Many efforts [26-29] have been devoted to exploring how reference points are formed since the prospect theory and other theories that involve reference dependence do not explain it. For instance, Tversky and Kahneman [27] pointed out that reference points may be influenced by the social norm and comparison. Recently, Baillon et al. [29] summarized six reference point rules and conducted comparative analysis through experiments. Although the rules for forming reference points are diverse, according to different information sources, reference points can be divided into endogenous reference points and exogenous reference points [30]. The information source of endogenous reference points usually comes from an individual own experience or memory, while reference points generated by other information are exogenous reference points. In addition, the existing economic studies [31-33] have demonstrated that most of the reference points have a common feature, namely, adaptability. Specifically, a new reference point may be a function of the previous reference point and information, which has been verified in empirical studies [34].

Collective behavior in human societies have turned out to be remarkably predictable [35]. In this paper, we propose a prospect theory based evolutionary vaccination game, of which the diagram is shown in Figure 1, to analyze and predict collective vaccination behavior against epidemics under the influence of evolving perception. Psychological perceptual change captured by the variable reference point is incorporated into the process of decision-making and strategy updating. Considering the diversity of the reference point, combined with the endogenous and exogenous ideas, we present two types of reference points, that is, the endogenous variable reference point and the endogenous-exogenous variable reference point, respectively. In order to evaluate the role of evolving perception on individual vaccination decision-making against epidemics, we compare the fraction of vaccinated individuals as well as infected individuals under variable

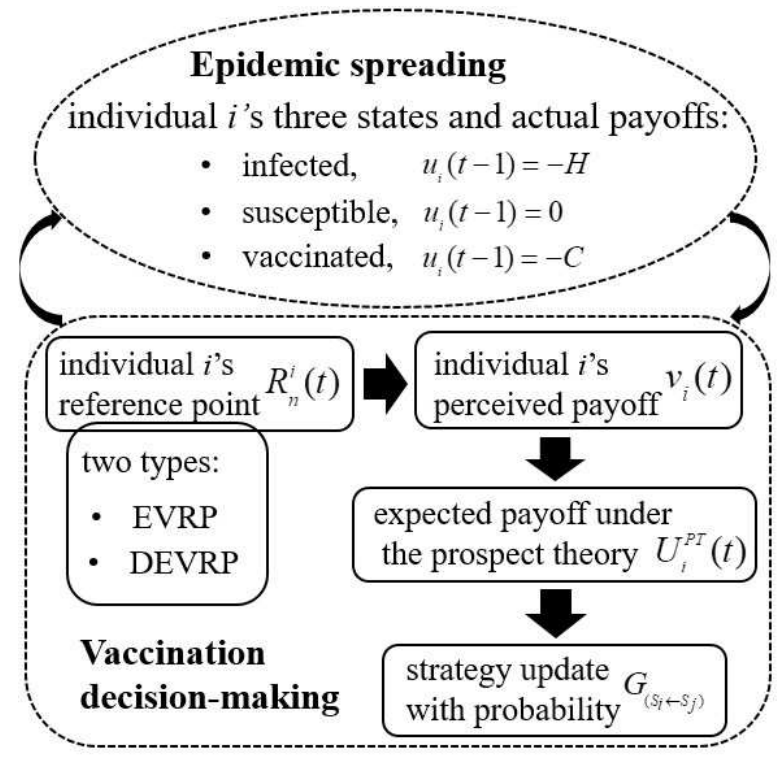

Fig. 1. The diagram of the evolutionary vaccination game.

reference points with those under the expected utility theory and the fixed reference point. The fixed reference point refers to the situation where each individual has the same and fixed reference point under the external control. Furthermore, we develop a mixed-reference-point mechanism based on the degree distribution, and numerical simulations on different heterogeneous networks show its effectiveness in reducing the final epidemic size.

\section{The evolutionary vaccination game with evolving perception}

\subsection{SIS epidemic model}

In order to explore the impact of evolving perception on vaccination behavior, we assume that the individual immunity only comes from vaccination. We utilize the SIS model to describe the epidemic spreading process (such as influenza). At each time step, an infected individual infects a susceptible individual with probability $\beta$, and the infected individual recovers with probability $\mu$. At time $t$, a susceptible individual $i$ has $k_{i}^{i n f}(t)$ infected neighbors. The infection probability of individual $i$ is

$$
p_{i}(t)=1-(1-\beta)^{k_{i}^{i n f}(t)} .
$$

\subsection{Vaccination game model under the prospect theory}

In the vaccination game, an individual unilaterally decides whether or not to be vaccinated. The timing of individual vaccination-making is different for different types of epidemics. For instance, individuals will make their vaccination decisions before the outbreak of the seasonal epidemics. In this paper, we consider some emergent diseases (such as H1N1), in this case, the vaccination decision-making of an individual may occur at any time 
of the epidemic spreading process. The time scales of epidemic dynamics and the vaccination updating dynamics are the same. The decision depends on not only one's tradeoff between the vaccination $\operatorname{cost} C$ and the infection cost $H$, but also the strategies of other individuals. We assume that individuals who choose vaccination can be fully protected, while others have a risk of being infected. Denote the infection probability of an unvaccinated individual as $p$. Classical game theory assumes that individuals are fully rational, and their decision-making are based on the expected utility theory. The expected payoff of a rational individual $i$ under the expected utility theory is

$$
U_{i}^{E U T}=-C m_{s}-\left(1-m_{s}\right) H p .
$$

If individual $i$ chooses to be vaccinated, the coefficient $m_{s}=1$, otherwise, $m_{s}=0$.

However, individual real-life behavior affected by subjective perception tends to deviate from the rational principle of the expected utility theory, showing bounded rationality. For instance, an unvaccinated individual perceives his payoff differently from its true value. In this paper, we introduce the prospect theory, which accounts for individual subjective perception under risk and uncertainty, into the vaccination game and get the prospecttheoretic expected payoff of an individual.

Individual subjective perception with respect to payoffs is captured by the framing effect of the prospect theory. An individual perceives a payoff either as a gain or a loss based on the reference point. A payoff is perceived as a gain when it is larger than the reference point, while it is regarded as a loss when it is smaller than the reference point. Generally, the reference point differs among individuals and changes from time to time. Therefore, we use the variable reference point, denoted by $R_{n}^{i}(t)$, to characterize individual evolving perception.

How the reference point is formed? Behavioral economics researches $[36,37]$ show that the sunk cost (incurred and irrecoverable cost) affects individual decision, and if the sunk cost is not taken into account, individual actual behavior may not be predicted. Therefore, in this paper, based on the adaptability $[32,33]$ and diversity [38] characteristics of the reference point, combined with endogenous and exogenous ideas, we propose two types of reference points, the endogenous variable reference point and the endogenous-exogenous variable reference point. These corresponding generation rules are as below.

1. The endogenous variable reference point (EVRP) Considering that reference points may be related to individual experience or memory (with respect to past payoffs), we present the weighted average of the perceived payoff and actual payoff of individual previous strategy as the endogenous variable reference point. The endogenous variable reference point of individual $i$ at time $t$ is expressed as

$$
R_{n}^{1 i}(t)=\frac{\phi v_{i}(t-1)+(1-\phi) u_{i}(t-1)}{2},
$$

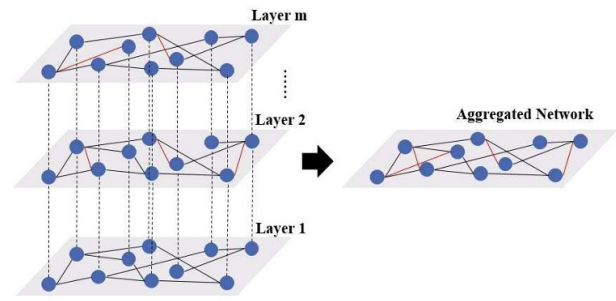

Fig. 2. A multilayer network and its corresponding aggregated network. Layer 1 represents an epidemic propagation (contact) network.

where $v_{i}(t-1)$ and $u_{i}(t-1)$ are perceived and actual payoffs of the previous strategy, respectively, and $\phi \in$ $[0,1]$ is the weighting coefficient.

2. The endogenous-exogenous variable reference point (DEVRP)

In reality, individuals are simultaneously in various networks, such as an epidemic propagation network and different online social networks. We propose an endogenous-exogenous variable reference point since the reference point of an individual may be affected by not only his own past payoff but also his neighbor's payoff. Considering that an individual may have different neighbors in different networks (layers), we obtain an aggregated network by integrating all networks, as shown in Figure 2. The endogenousexogenous variable reference point is defined as the weighted average of the previous strategies' actual payoffs of both individual $i$ and his neighbor $j$, who is randomly chosen in the aggregated network, denoted by $R_{n}^{2 i}(t)$.

$$
R_{n}^{2 i}(t)=\frac{\pi u_{i}(t-1)+(1-\pi) u_{j}(t-1)}{2}
$$

where $u_{i}$ and $u_{j}$ are the previous strategy's actual payoffs of individual $i$ and $j$, respectively, and $\pi \in$ $[0,1]$ is the weighting coefficient.

In addition to the reference dependence preferences, individuals are more sensitive to losses than gains. The value function proposed by Tversky and Kahneman [27] is given by,

$$
v_{i}=\left\{\begin{array}{cc}
\left(u_{i}-R_{n}^{i}\right)^{\alpha} & \text { if } u_{i} \geq R_{n}^{i} \\
-\lambda\left(R_{n}^{i}-u_{i}\right)^{\alpha} & \text { if } u_{i}<R_{n}^{i}
\end{array}\right.
$$

where $R_{n}^{i}$ is the reference point of individual $i, \lambda \geq 1$ and $\alpha \in(0,1]$ are the loss multiplier and sensitivity coefficient, respectively, $u_{i}$ and $v_{i}$ are the actual and perceived payoffs of individual $i$, respectively. If individual $i$ is unvaccinated and infected, the payoff $u_{i}=-H$, otherwise, $u_{i}=0$.

Let $H=1$ and $\alpha=1$. If individual $i$ chooses to be vaccinated at time $t$, his expected payoff is $-C$, otherwise, his expected payoff under the prospect theory denoted by 


$$
\begin{aligned}
& U_{i}^{P T}(t) \text { is } \\
& U_{i}^{P T}(t)=\left\{\begin{array}{l}
-p_{i}(t)-R_{n}^{i}(t) \quad \text { if } R_{n}^{i}(t)<-1, \\
\left((1-\lambda) p_{i}(t)-1\right) R_{n}^{i}(t)-\lambda p_{i}(t) \\
\text { if }-1 \leq R_{n}^{i}(t) \leq 0 \\
-\lambda\left(p_{i}(t)+R_{n}^{i}(t)\right) \quad \text { if } \quad R_{n}^{i}(t)>0
\end{array}\right.
\end{aligned}
$$

where $p_{i}(t)$ and $R_{n}^{i}(t)$ are the infection probability and variable reference point of individual $i$ at time $t$, respectively.

Individuals update their vaccination strategies based on the pairwise comparison rule in the epidemic spreading process. At each time step, individual $i$ randomly chooses neighbor $j$ in the epidemic propagation network, and imitates the strategy of individual $j$ with probability $G_{\left(S_{i} \leftarrow S_{j}\right)}$,

$$
G_{\left(S_{i} \leftarrow S_{j}\right)}=\frac{1}{1+\exp \left[-\kappa\left(U_{j}^{P T}-U_{i}^{P T}\right)\right]},
$$

where $\kappa$ is the selection intensity, $S_{i}$ and $U_{i}^{P T}$ are the strategy and prospect-theoretic expected payoff of individual $i$, respectively. Previous researches $[39,40]$ have studied the effects of different update rules by means of the Fermi function [41]. However, psychological perception has not been taking into account. In this paper, we incorporate individual subjective perception into the process of decision-making and strategy update using a prospect theory framework, and explore the role of evolving perception on individual vaccination decision-making and epidemic control.

\section{Numerical simulations}

In order to study the collective vaccination behavior under the influence of evolving perception, we perform simulations on several heterogeneous networks (BA scale-free network with $m=3$, contact network, social network and Facebook page network $[42,43])$. The fractions of vaccinated individuals and infected individuals are initially set to 0.1 , the recovery probability $\mu=0.1$ and $\kappa=10$. The evolutionary vaccination game is iterated in time by a Monte Carlo method. The pairwise comparison process is repeated $N$ times to guarantee that each individual has a chance to update one's strategy. The Monte Carlo simulations are averaged by 50 realizations.

\subsection{Performance analysis of the variable reference point}

To explore the effects of reference points generated by two different rules on vaccination decision making, we perform simulations on a BA scale-free network and a real network, respectively. The whole evolutionary game process is evolved for 200 time steps $\left(t_{\max }=200\right)$. For the convenience of comparison, we take the same weighting coefficient under different formulation rules of the reference point. Let $\phi=\pi=0.1$.

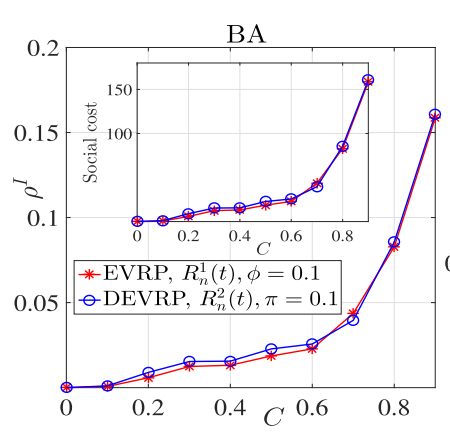

(a)

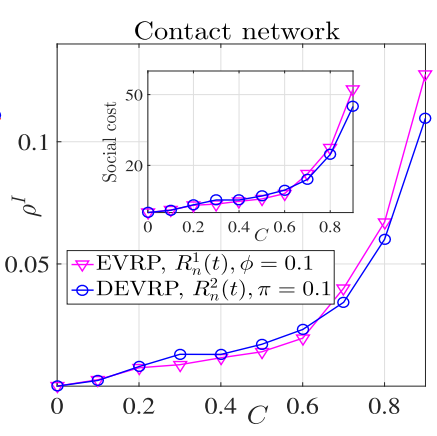

(b)
Fig. 3. The comparisons of final fraction of infected individuals $\rho^{I}$ (main), and social cost (inset), under the EVRP and DEVRP in (a) BA scale-free network $(N=1000)$ and (b) contact network $(N=410)$. Parameters: $\beta=0.6, \mu=0.1, \lambda=2$ and $t_{\max }=200$.

Figure 3 shows the comparative results between the EVRP and the DEVRP. Denote the fraction of infected individuals in a network as $\rho^{I}$. We find that when the vaccination $\operatorname{cost} C$ is relatively small $(C<0.6)$, the final fraction of infected individuals $\rho^{I}$ under the EVRP is always less than that under the DEVRP, regardless of the network topology. That is, the vaccination decisionmaking based on individuals' own experience is more advantageous to epidemic control when the vaccination cost is relatively small. When the vaccination $\operatorname{cost} C$ is relatively large, the final fraction of infected individuals $\rho^{I}$ under the EVRP is larger than or equal to that under the DEVRP. In this case, provide as much information (strategies or payoffs) as possible about their neighbors to the individual, so that an individual can make vaccination decision based on the common experiences of himself and his neighbors, which is more conducive to controlling the epidemic spreading, when the vaccination cost is relatively large. Therefore, we conclude that the performance of evolving perception on reducing the epidemic size depends on the vaccination cost.

\subsection{Vaccination cost threshold under pure reference point}

In order to further illustrate the impact of evolving perception on epidemic control, we compare the fraction of infected individuals under the variable reference point with that under the expected utility theory (EUT) and the fixed reference point (FRP). Moreover, we refer to the case where all individuals have a fixed reference point or variable reference points as the case of pure reference point.

Figure 4 shows the fractions of vaccinated individuals $x$ and infected individuals $\rho^{I}$ as a function of time $t$ under the EVRP, EUT and FRP in a BA scale-free network and a Facebook page network, respectively. Whether it's under the EVRP, EUT or FRP, the time $t_{s}$ for the system to reach the steady state is basically the same. For instance, $t_{s}=15$ in the BA scale-free network (see Figs. 4a and 4b). Figure 5 shows the comparison between the EVRP, EUT 


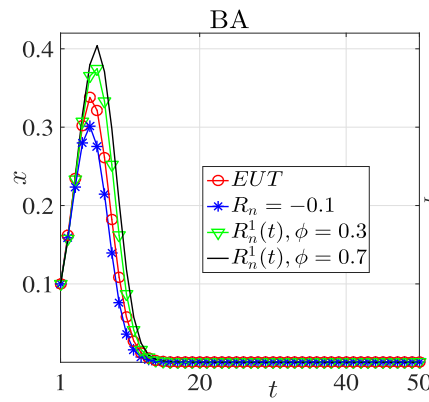

(a)

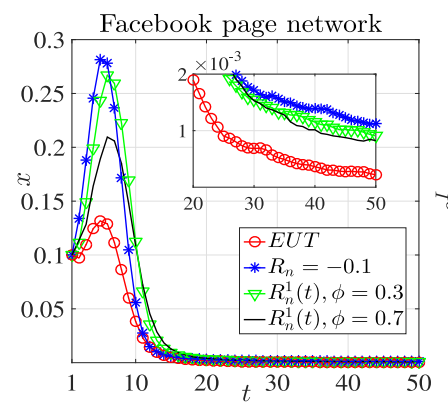

(c)

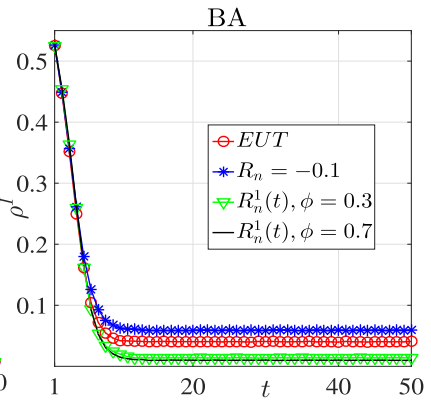

(b)

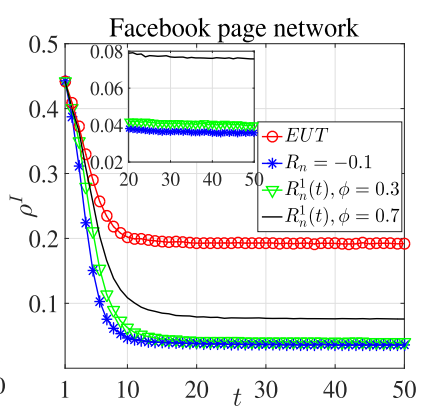

(d)
Fig. 4. The fractions of vaccinated individuals $x$ and infected individuals $\rho^{I}$ as a function of time $t$ under the EVRP, EUT and FRP in (a) BA scale-free network $(N=3000)$ and (b) Facebook page network $(N=5908)$. Parameters: $\beta=0.6, \mu=$ $0.1, \lambda=2$ and $t_{\max }=50$.

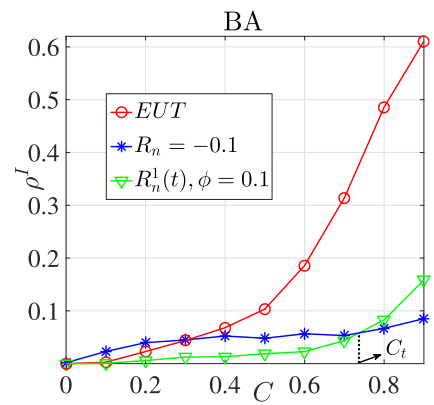

(a)

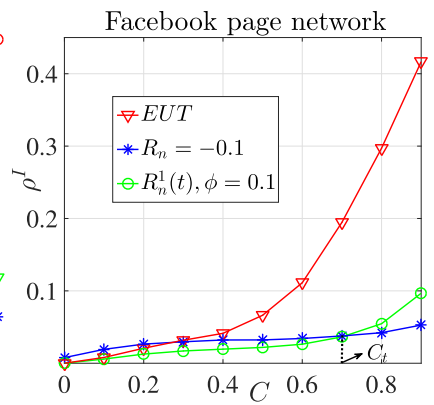

(b)
Fig. 5. The comparison of final fractions of infected individuals $\rho^{I}$ under the EVRP, EUT and FRP in (a) BA scale-free network $(N=3000)$ and (b) Facebook page network $(N=5908)$. Parameters: $\beta=0.6, \mu=0.1, \lambda=2$ and $t_{\max }=200$.

and FRP. We find that the final fraction of infected individuals $\rho^{I}$ under the EVRP is always less than that under the EUT, regardless of the vaccination cost. That is, the vaccination decision-making of an individual who takes into account the influence of psychological perception is more beneficial to the control of epidemic transmission, compared with the decision made by a completely rational individual.

In terms of the EVRP and FRP, comparative result depends on the vaccination cost. As illustrated in Figure 5, when the vaccination $\operatorname{cost} C$ is less than a certain threshold $C_{t}$, the final fraction of infected individuals $\rho^{I}$ under

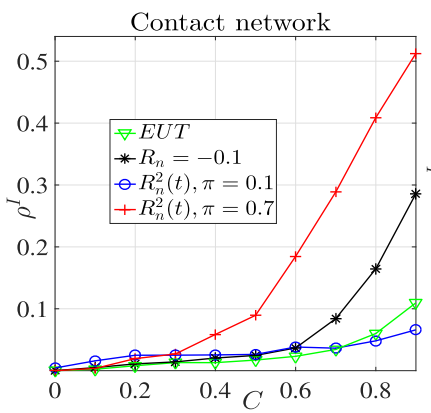

(a)

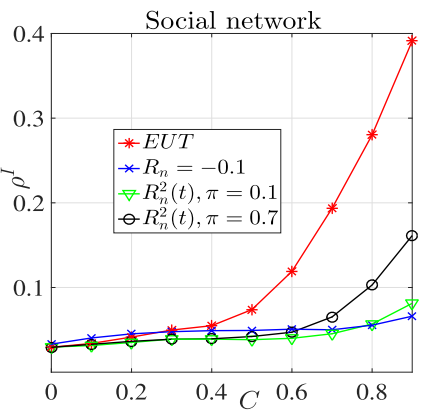

(b)
Fig. 6. The comparison of final fractions of infected individuals $\rho^{I}$ under the DEVRP, EUT and FRP in (a) contact network $(N=410)$ and (b) social network $(N=2426)$. Parameters: $\beta=0.6, \mu=0.1, \lambda=2$ and $t_{\max }=200$.

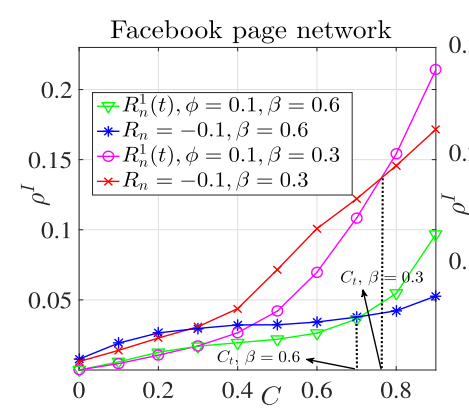

(a)

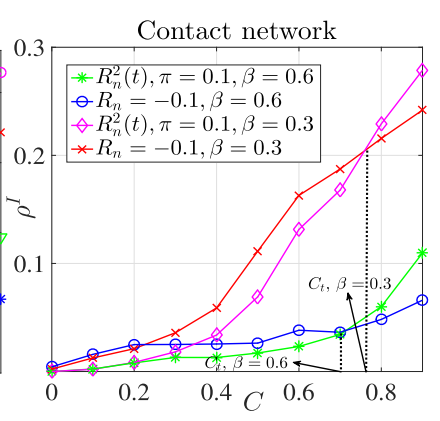

(b)
Fig. 7. The vaccination cost threshold $C_{t}$ under different infection rates $\beta$. (a) EVRP; (b) DEVRP. Parameters: $\mu=0.1$, $\lambda=2$ and $t_{\max }=200$.

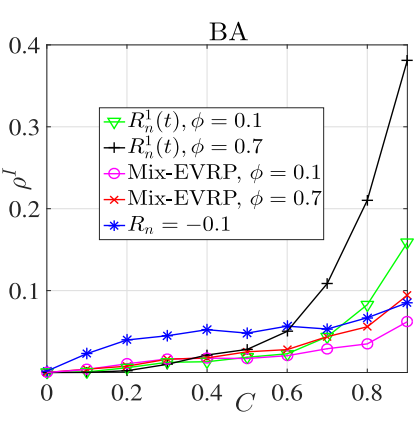

(a)

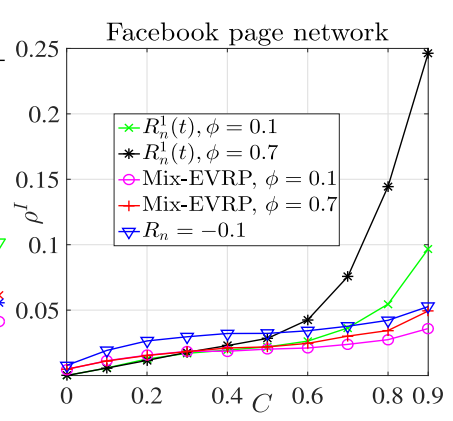

(b)
Fig. 8. The comparison of final fractions of infected individuals $\rho^{I}$ under the Mix-EVRP, EVRP and FRP in (a) BA scale-free network $(N=1000)$ and (b) Facebook page network $(N=$ 5908). Parameters: $\beta=0.6, \mu=0.1, \lambda=2$ and $t_{\max }=200$.

the EVRP is less than that under the $\operatorname{FRP}\left(R_{n}=-0.1\right)$. When the vaccination cost $C$ exceeds the threshold $C_{t}$, the final fraction of infected individuals $\rho^{I}$ under the FRP is the smaller. The similar conclusion can be obtained under the DEVRP in Figure 6. Although the FRP helps reduce the final fraction of infected individuals when the vaccination cost $C$ exceeds the threshold $C_{t}$, implementing the FRP (each individual has a same and fixed reference point) usually requires external control. 

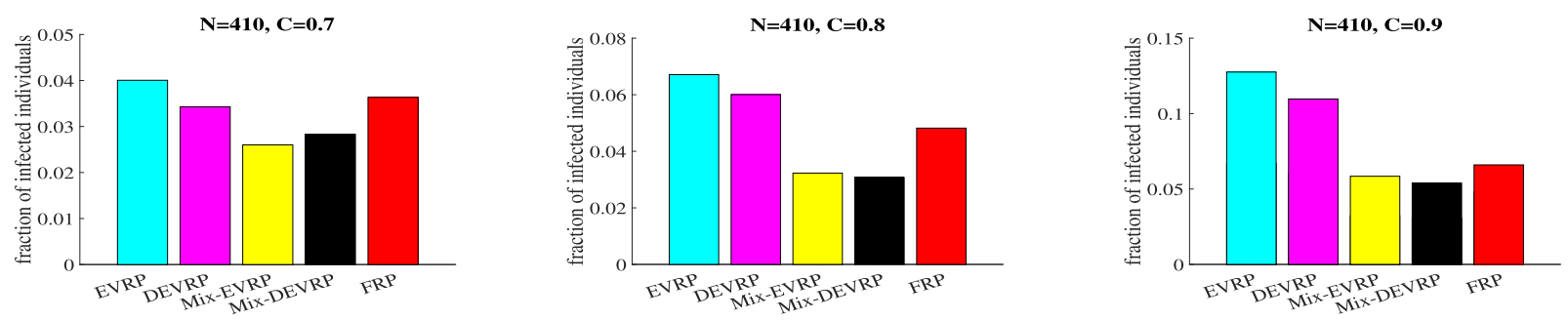

(a)
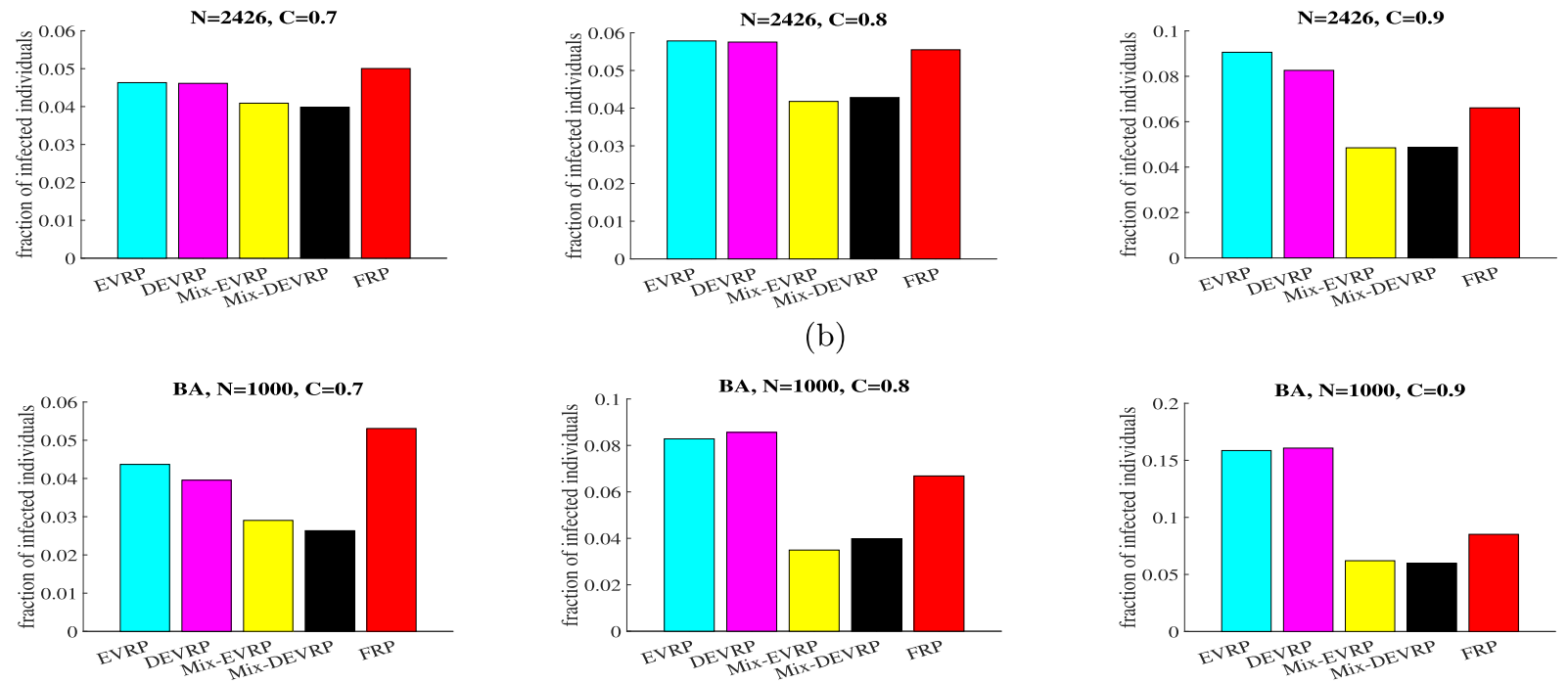

(c)

Fig. 9. The comparison between pure reference point and mixed reference point under large vaccination costs. The blue, pink, yellow, black and red parts in each bar chart correspond to the fractions of infected individuals under the EVRP, DEVRP, Mix-EVRP, Mix-DEVRP and FRP, respectively.

We further explore the impact factor of the vaccination cost threshold since there exists a vaccination cost threshold in the comparison between the EVRP (DEVRP) and FRP. Figure 7a shows the comparative result between the EVRP and FRP with different infection rates $\beta$. As illustrated in Figure 7, the vaccination cost threshold increases with the decrease of infection rate $\beta$, it doesn't matter whether it is under the EVRP or DEVRP.

\subsection{Performance analysis of the mixed reference point}

From the result of comparison between the EVRP (DEVRP) and FRP, we find that external control is needed to fix the reference point to reduce the final epidemic size $\rho^{I}$ as much as possible, even if the vaccination $\operatorname{cost} C$ is small, when the infection rate $\beta$ is large. Therefore, a new mechanism is needed since fixing reference points of all individuals in a network is difficult to achieve in reality.

We propose a new mixed-reference-point mechanism, in which individuals with smaller degrees have fixed reference points, and those with larger degrees have variable reference points. For instance, individual $i$ has an endogenous variable reference point with probability $k_{i} / k_{\max }$, and has a fixed reference point with probability $1-k_{i} / k_{\max }$. Corresponding to the two variable reference points (EVRP and DEVRP), we define two types of mixed-referencepoint: Mix-EVRP and Mix-DEVRP.

To illustrate the effectiveness of the mixed reference point in controlling the epidemic spreading, we compare the final fraction of infected individuals $\rho^{I}$ under the MixEVRP, EVRP and FRP. In Figure 8, we find that the final fraction of infected individuals $\rho^{I}$ under the Mix-EVRP is the smallest when the vaccination $\operatorname{cost} C$ exceeds a certain value, regardless of the network topology.

We compare the final fraction of infected individuals $\rho^{I}$ under pure reference point (EVRP, DEVRP and FRP) and mixed reference point to further study the impact of mixed reference point on epidemic control when the vaccination cost $C$ is large. As illustrated in Figure 9, the final fractions of infected individuals $\rho^{I}$ under mixed reference points (the yellow and black parts of the bar chart) is always less than those under pure reference points (the blue and pink parts of the bar chart), regardless of the network topology and vaccination cost $C$. In other words, both Mix-EVRP and Mix-DEVRP can effectively reduce the final epidemic size at a large vaccination cost, compared with pure reference points. Different from the finding that hub nodes play a leading role in implementing imitation strategy to suppress epidemic spreading in [44], we find that it is more critical to implement the control strategy for individuals with smaller degrees to fix their reference points. 


\section{Conclusion}

Taking into account the realistic characteristics of individual psychological cognition, we have constructed a prospect theory based evolutionary vaccination game model, analyzed and predicted collective vaccination behavior against epidemics under the influence of evolving perception. In this paper, perceptual change is captured by the variable reference point. We find that the final fraction of infected individuals under variable reference point is always less than that under the expected utility theory, which indicates the advantage of evolving perception in promoting vaccination and helping the epidemic control. Moreover, compared with the fixed reference point that requires external control to achieve, the performance of variable reference points in epidemic control depends on the vaccination cost, showing the double-edge sword effect. Motivated by this effect, we have proposed a new mixed-reference-point mechanism based on the degree distribution, which can effectively reduce the final epidemic size, regardless of the network topology, and alleviate the problem that epidemic transmission is difficult to control at a large vaccination cost. Our findings uncover the role of psychological characteristics of individuals on vaccination taking-up, and may provide a guidance for managers in implementing policy management and guiding public anti-epidemic behavior.

This work was partly supported by the National Natural Science Foundation of China (No. 61751303, No. 71731004) and the Natural Science Fund of Distinguished Young Scholarship of China (No. 61425019).

\section{Author contribution statement}

All authors listed have made a substantial, direct and intellectual contribution to the work, and approved it for publication.

Publisher's Note The EPJ Publishers remain neutral with regard to jurisdictional claims in published maps and institutional affiliations.

\section{References}

1. N. Zhu, D. Zhang, W. Wang, X. Li, B. Yang, J. Song, X. Zhao, B. Huang, W. Shi, R. Lu et al., N. Engl. J. Med. 382, 727 (2020)

2. I.R. Tizard, Vaccine 38, 5123 (2020)

3. C.T. Bauch, A.P. Galvani, D.J. Earn, Proc. Natl. Acad. Sci. U.S.A. 100, 10564 (2003)

4. C.T. Bauch, D.J. Earn, Proc. Natl. Acad. Sci. U.S.A. 101, 13391 (2004)

5. Z. Wang, C.T. Bauch, S. Bhattacharyya, A. Donofrio, P. Manfredi, M. Perc, N. Perra, M. Salathe, D. Zhao, Phys. Rep. 664, 1 (2016)

6. M. Perc, J.J. Jordan, D.G. Rand, Z. Wang, S. Boccaletti, A. Szolnoki, Phys. Rep. 687, 1 (2017)
7. C.T. Bauch, Proc. R. Soc. London B 272, 1669 (2005)

8. F. Fu, D.I. Rosenbloom, L. Wang, M.A. Nowak, Proc. R. Soc. London B 278, 42 (2011)

9. H. Zhang, F. Fu, W. Zhang, B. Wang, Physica A 391, 4807 (2012)

10. Z.X. Wu, H.F. Zhang, Europhys. Lett. 104, 10002 (2013)

11. A. Grabowski, Eur. Phys. J. B 87, 146 (2014)

12. B. Buonomo, Ric. Mater., https://doi.org/10.1007/ s11587-020-00506-8

13. T. Oraby, C.T. Bauch, Sci. Rep. 5, 10724 (2015)

14. D.G. Rand, Psychol. Sci. 27, 1192 (2016)

15. A. Bear, D.G. Rand, Proc. Natl. Acad. Sci. U.S.A. 113, $936(2016)$

16. H.A. Simon, Q. J. Econ. 69, 99 (1955)

17. A.P. Galvani, T.C. Reluga, G.B. Chapman, Proc. Natl. Acad. Sci. U.S.A. 104, 5692 (2007)

18. X.J. Li, C. Li, X. Li, IEEE Trans. Netw. Sci. Eng. 5, 326 (2018)

19. D. Ellsberg, Q. J. Econ. 75, 643 (1961)

20. D. Kahneman, A. Tversky, Econometrica 47, 263 (1979)

21. C. Jie, L. Prashanth, M. Fu, S. Marcus, C. Szepesvári, IEEE Trans. Automat. Control 63, 2867 (2018)

22. A. Thanou, E.E. Tsiropoulou, S. Papavassiliou, IEEE Trans. Comput. Soc. Syst. 6, 135 (2019)

23. A.R. Hota, S. Sundaram, IEEE Trans. Control Netw. 6, $1461(2019)$

24. X.J. Li, X. Li, IEEE Trans. Comput. Soc. Syst. 7, 329 (2019)

25. M. Rabin, Econometrica 68, 1281 (2000)

26. S. Siegel, Psychol. Rev. 64, 253 (1957)

27. A. Tversky, D. Kahneman, Q. J. Econ. 106, 1039 (1991)

28. M. Wu, T. Bai, S.X. Zhu, Omega 81, 99 (2018)

29. A. Baillon, H. Bleichrodt, V. Spinu, Manage. Sci. 66, 93 (2020)

30. R. Sugden, J. Econ. Theory 111, 172 (2003)

31. J.M. Lattin, R.E. Bucklin, J. Marketing Res. 26, 299 (1989)

32. D.R. Bell, J.M. Lattin, Marketing Sci. 19, 185 (2000)

33. M. Baucells, R.K. Sarin, Manage. Sci. 56, 286 (2010)

34. T.K. Lant, Manage. Sci. 38, 623 (1992)

35. M. Perc, Sci. Rep. 9, 1 (2019)

36. H.R. Arkes, C. Blumer, Organ. Behav. Hum. Dec. 35, 124 (1985)

37. H.R. Arkes, L. Hutzel, J. Behav. Decis. Making 13, 295 (2000)

38. M. Baucells, M. Weber, F. Welfens, Manage. Sci. 57, 506 (2011)

39. E. Fukuda, S. Kokubo, J. Tanimoto, Z. Wang, A. Hagishima, N. Ikegaya, Chaos Solitons Fractals 68, 1 (2014)

40. X. Chen, L. Wang, Phys. Rev. E 77, 017103 (2008)

41. G. Szabó, C. Tőke, Phys. Rev. E 58, 69 (1998)

42. R.A. Rossi, N.K. Ahmed, The Network Data Repository with Interactive Graph Analytics and Visualization (Austin, Texas, 2015)

43. B. Rozemberczki, R. Davies, R. Sarkar, C. Sutton, GEMSEC: Graph Embedding with Self Clustering (Vancouver, British Columbia, 2019)

44. H.F. Zhang, Z. Wang, IEEE Trans. Circuits Syst. II Express Briefs, https://doi.org/10.1109/TCSII.2019. 2938775 